\section{Autophagic degradation determines the fate of T315I-mutated BCR-ABL protein}

Marked clinical improvement has been achieved by the use of BCR-ABL-tyrosine kinase inhibitors; however, mutations in BCR-ABL are among the most common causes of resistance to these inhibitors.

We previously found that AIC-47, a novel autophagy inducer, suppresses the expression of $\mathrm{BCR}-\mathrm{ABL},{ }^{1,2}$ suggesting that AIC-47 could affect the expression of mutated BCR-ABL. So, in the present study we estimated the effects of AIC-47 on BCR-ABL mutant cells by using BCR-ABL-expressing Baf3 cells ${ }^{3}$ and TCCY cells. ${ }^{4}$ AIC-47 was found to suppress the phosphorylation and expression of wildtype (WT)-BCR-ABL; however, T315I-BCR$\mathrm{ABL}$ expression was hardly suppressed by AIC-47 (Figure $1 \mathrm{~A}$ and Online Supplementary Figure S2A). In Baf3p210 cells harboring other BCR-ABL mutations (M351T or Y253F), the suppression was observed (Online Supplementary Figure S2B), suggesting that the regulation of T315I-BCR-ABL generation and/or degradation was different from that of other types of BCR-ABL. We then investigated the generation and degradation of T315IBCR-ABL. Our previous studies showed that AIC-47 suppresses transcription of $B C R-A B L .{ }^{1}$ The level of expression of $B C R-A B L$ mRNA was decreased after treatment with AIC-47 in both WT- and T315I-BCR-ABL-harboring cells (Online Supplementary Figure S3A). AIC-47 also decreased $B C R-A B L$ mRNA levels in primary cells from patients (Online Supplementary Figure S3B). At steady state, the decay of $B C R-A B L$ mRNA was not significantly different between WT- and T315I-BCR-ABL cells (Online Supplementary Figure S3C). Moreover, inhibition of translation of $B C R-A B L$ mRNA by cycloheximide or puromycin resulted in a delay in the elimination of T315I-BCR-ABL compared with that of WT-BCR-ABL (Figure 1B), suggesting that degradation contributed to the level of BCR-ABL protein expression. In cells, the majority of intracellular proteins are degraded by the ubiquitin-proteasome pathway and autophagy. We found that the autophagy inhibitor 3-methyladenine (3-MA) suppressed the degradation of both types of BCR-ABL; whereas the proteasome inhibitor MG-132 did not sup-
A
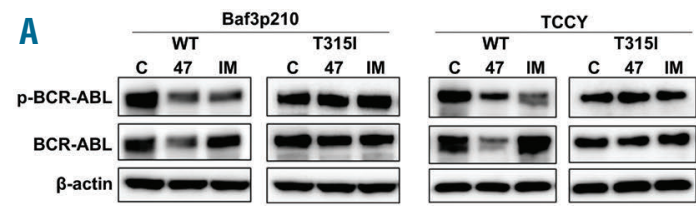

B
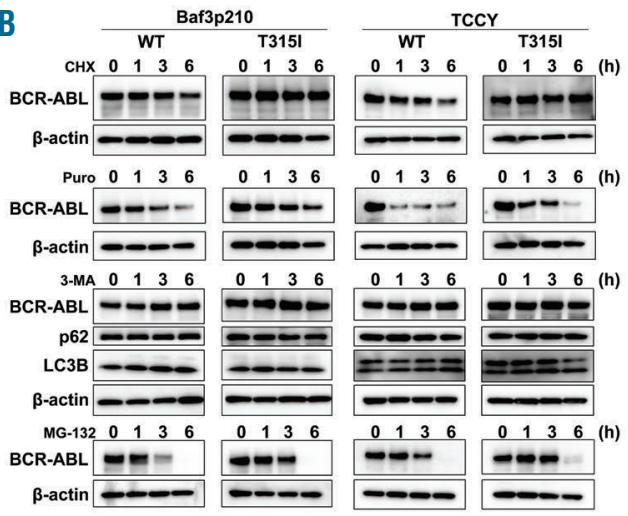

C
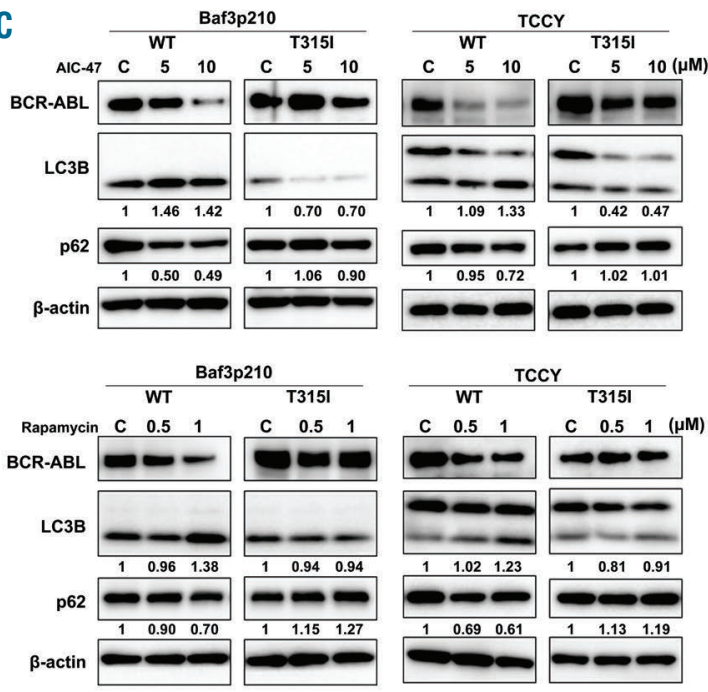

Baf3p210 WT

E

D

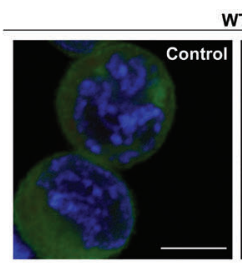

WT
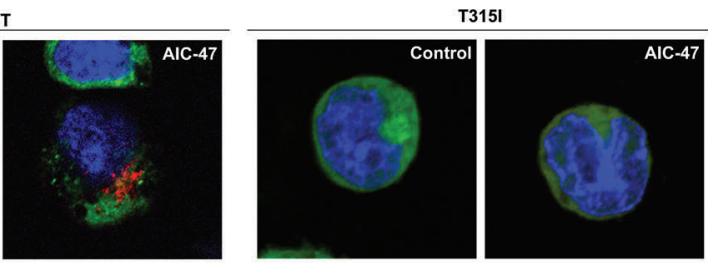

Figure 1. Impaired autophagic degradation increased the stability of T315I-BCR-ABL protein. (A) Effects of AIC-47 or imatinib on phosphorylation and expression of WT- and T315I-BCR-ABL in Baf3p210 and TCCY cells. The cells were treated with dimethylsulfoxide (DMSO; Control: C), AIC-47 (47; $10 \mu \mathrm{M})$ or imatinib (IM; $0.25 \mu \mathrm{M})$ for $48 \mathrm{~h}$, followed by western blotting analysis. (B) Time-dependent expression of BCR-ABL after the treatments. To inhibit generation of BCR-ABL proteins, Baf3p210 and TCCY cells were treated with cycloheximide (CHX; $2.5 \mu \mathrm{g} / \mathrm{mL}$ ) or puromycin (Puro; $1 \mathrm{pg} / \mathrm{mL})$. To inhibit degradation of BCR-ABL proteins, an autophagy inhibitor 3-methyladenine (3-MA; $100 \mu \mathrm{M})$ and a proteasome inhibitor MG-132 (10 $\mu \mathrm{M})$ were used. The levels of expression of BCR-ABL, p62, and LC3B proteins were determined by western blotting analysis. (C) Effect of AIC-47 on autophagy flux and BCR-ABL expression. Expression of BCR-ABL and p62, and conversion of LC3B, in Baf3p210 and TCCY cells treated with AIC-47 ( 5 or $10 \mu \mathrm{M}$ ) for $24 \mathrm{~h}$ were examined by western blotting analysis. The numbers below p62 and LC3B indicate each band density relative to that of the Control (taken as "1"), whose values were determined by densitometry. (D) Distribution of ABL and the autophagy marker LC3 in AIC-47-treated cells. Baf3p210 cells were incubated with AIC-47 (5 $\mu \mathrm{M})$ for $12 \mathrm{~h}$ and then co-immunostained with c-ABL and LC3 antibodies. The cells were viewed with a LSM710 confocal laser scanning microscope. Representative images are shown. ABL is stained green; and the LC3 is stained red. Nuclei appear in blue. (E) Effect of the autophagy inhibitors 3-MA and chloroquine (CQ) on BCR-ABL expression. WT-BCR-ABL-harboring cells were pretreated with 3-MA $(100 \mu \mathrm{M})$ or CQ $(1 \mu \mathrm{M})$ for $4 \mathrm{~h}$, and then treated with DMSO or AIC-47 $(5 \mu \mathrm{M})$ for $24 \mathrm{~h}$. The expression levels of BCR-ABL and p62 were examined by western blotting analysis. 
press their degradation (Figure 1B). These findings indicate that autophagic degradation was closely associated with the fate of BCR-ABL protein.

Next, we investigated the induction of autophagy in AIC-47-treated cells. Interestingly, AIC-47 treatment resulted in several findings of autophagy, including: (i) LC3 lipidation, as indicated by the conversion of LC3B I to II; (ii) induction of Atg proteins; and (iii) degradation of p62 in the cells with WT-BCR-ABL, but not in those with T315I-BCR-ABL (Figure 1C and Online Supplementary Figure S4). The induction of autophagy occurs preferentially in the G1 and S phases of the cell cycle. ${ }^{5}$ The number of WT-BCR-ABL cells in the G0/G1 phase increased after treatment with AIC-47, whereas a change in the profile of the cell-cycle was barely observed in T315I-
BCR-ABL cells (Online Supplementary Figure S1C). The impaired autophagy in the T315I-mutated cells was also observed in rapamycin-treated cells (Figure 1C and Online Supplementary Figure S5). After AIC-47 treatment, LC3 was punctate and co-localized with BCR-ABL in WTBCR-ABL cells, indicating that BCR-ABL had been degraded in autophagosomes, whereas LC3 puncta were barely observed in T315I-BCR-ABL cells (Figure 1D). To further investigate the AIC-47-induced autophagic degradation of BCR-ABL, we inhibited autophagy flux in WTBCR-ABL cells by using 3-MA or chloroquine. The pretreatment with these autophagy inhibitors partly abolished the decreased expression of BCR-ABL induced by AIC-47 (Figure 1E). Based on these results, we speculated that dysregulated autophagic degradation contributed to
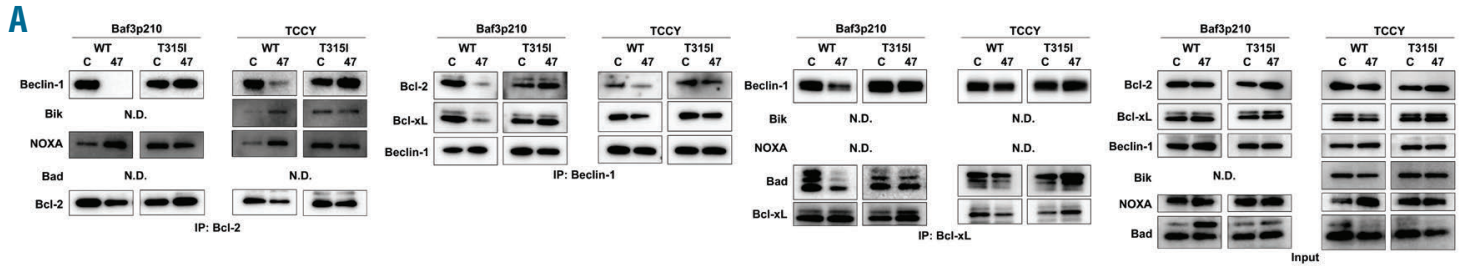

B

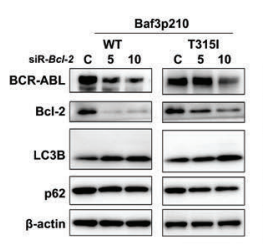

E

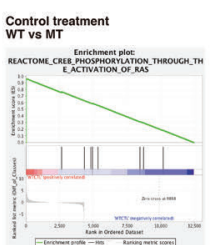

AIC treatment
WT vs MT

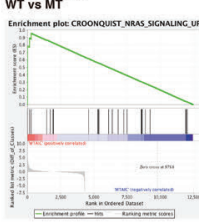

C

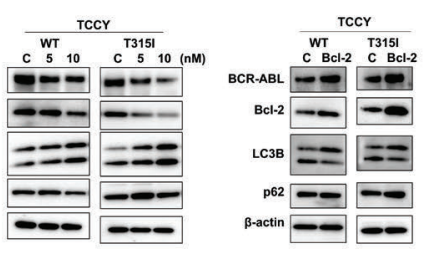

D

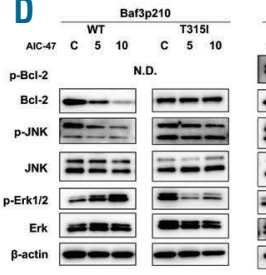

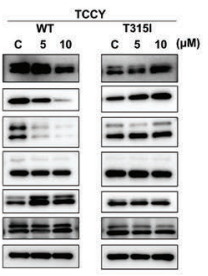

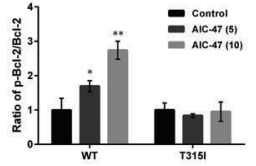

G
$\mathrm{F}$
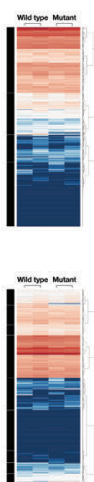

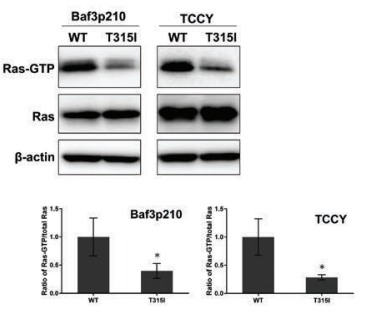

(1)

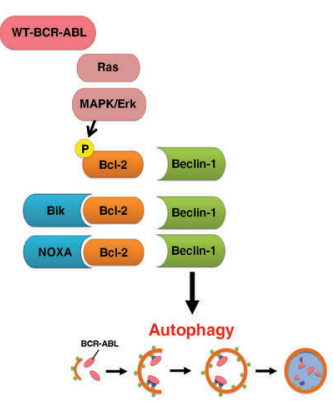

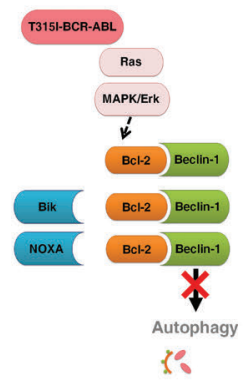

Figure 2. The dissociation of Bcl-2 from Beclin-1 was impaired in T315I-BCR-ABL-harboring cells. (A) Immunoprecipitation of Baf3p210 and TCCY cell lysates. Cells were treated with AIC-47 (5 $\mu \mathrm{M})$ for $24 \mathrm{~h}$. The expressed Bcl-2, Beclin-1 or Bcl-xL was pulled down by antibodies corresponding to each protein. The binding of each protein was detected by western blotting analysis. Anti-Bik antibody could not detect Bik of mouse origin. (B) Effects of silencing Bcl-2 on autophagy flux in Baf3p210 and TCCY cells. Cells were transfected with control RNA or siRNA for Bcl-2 (5 or $10 \mathrm{nM}$ ) for $72 \mathrm{~h}$; and then expression levels of BCR-ABL and p62, and the conversion of LC3B, were examined by western blotting analysis. (C) Effects of overexpression of Bcl-2 on autophagy flux in TCCY cells. Cells were transfected with pIRESneo-Bcl-2 vectors for $72 \mathrm{~h}$, and then expression levels of BCR-ABL and p62, and conversion of LC3B, were examined by western blotting analysis. (D) Effects of AIC-47 on the phosphorylation and expression of Bcl-2, JNK, and Erk in Baf3p210 and TCCY cells. The cells were treated with dimethylsulfoxide (DMSO; Control, C), or AIC-47 (5 or $10 \mu \mathrm{M}$ ) for $24 \mathrm{~h}$, followed by western blotting analysis (left panel). Anti-phospho-Bcl-2 antibody could not detect phosphorylated $\mathrm{Bcl}-2$ of mouse origin. The ratio of phosphorylated Bcl-2 was quantified by densitometry scanning and normalized to the expression level of Bcl-2 (right panel). Data are expressed as means \pm SD of three different experiments. $* P<0.05, * * * P<0.001$ versus Control (Student $t$-test). (E) The heat map shows all genes that were differentially expressed between WT-BCR-ABL cells (WT) and T315I-BCR-ABL cells (MT). Gene set enrichment analysis (GSEA) results of c2 reference gene sets revealed that Ras-related signatures were enriched in steady-state WT-BCR-ABL cells (false discovery rate $=0.138, P<0.001$ ). "NRAS signaling" signatures were also enriched in AIC-47-treated WT-BCR-ABL cells (false discovery rate $=0.039, P<0.001$ ). Horizontal white bars on the left of the heat maps indicate the genes within each gene set. GSEA was performed with GenePattern 2.0 software. (F) Activation of Ras in Baf3p210 and TCCY cells at steady state. GTP-bound Ras was estimated using a Ras assay kit. The level of Ras-GTP was quantified by densitometry scanning and normalized to the total level of Ras. (G) Schematic diagram of the difference in autophagic degradation between WT- and T315I-BCR-ABL. In T315I-BCR-ABL-harboring cells, the activation of Ras/MAPK signaling is less than that in WT-BCR-ABL-harboring cells, which reduces the phosphorylation of Bcl-2. The dissociation of Bcl-2 from Beclin-1 is thus impaired in T315I-BCR-ABL cells, resulting in impaired autophagic degradation of T315I-BCR-ABL 
the increased stability of T315I-BCR-ABL and might be one of the mechanisms of resistance to tyrosine kinase inhibitors.

In the autophagy network, Bcl-2 interacts with Beclin1 and contributes to the inhibition of autophagy flux. ${ }^{6,7}$ Treatment with Z36, another inducer of autophagy, which dissociates $\mathrm{Bcl}-2 / \mathrm{Bcl}-\mathrm{xL}$ and Beclin- $1,{ }^{8}$ resulted in induction of LC3 lipidation in WT- and T315I-BCR-ABL cells (Online Supplementary Figure S6). The expression level of BCR-ABL protein decreased after treatment with Z36 in both WT- and T315I-BCR-ABL cells (Online Supplementary Figure S6). These results suggest that the interaction between $\mathrm{Bcl}-2 / \mathrm{Bcl}-\mathrm{xL}$ and Beclin-1 is associated with the impaired autophagy in T315I-BCR-ABL cells. Thus, we examined the interaction of $\mathrm{Bcl}-2 / \mathrm{Bcl}-\mathrm{xL}$ with Beclin-1 in AIC-47-treated cells. After AIC-47 treatment, Bcl-2 clearly dissociated from Beclin-1 in WTBCR-ABL cells; whereas binding remained in the T315IBCR-ABL cells (Figure 2A). Bcl-xL slightly dissociated from Beclin-1 (Figure 2A). There are several different means to regulate the dissociation of $\mathrm{Bcl}-2$ from Beclin1 , including competitive displacement of the Beclin-1 $\mathrm{BH} 3$ domain by other Bcl-2 family proteins, or by MAPK (JNK and Erk)-mediated phosphorylation of Bcl-2. 6,7 The interaction with $\mathrm{BH} 3$-only proteins Bik or NOXA and Bcl-2 was increased after treatment with AIC-47 in WTBCR-ABL cells (Figure 2A). Binding with Bcl-2 and Bad was not observed (Figure 2A), suggesting that Bik and NOXA bound to the $\mathrm{BH} 3$-binding groove of $\mathrm{Bcl}-2$ and disrupted the interaction between Beclin-1 and Bcl-2. $\mathrm{Bcl}-\mathrm{xL}$ bound Bad; however, the interaction was slightly decreased (Figure 2A). An interaction between Bik or NOXA and Bcl-xL was not observed (Figure 2A). Based on these data, we speculated that $\mathrm{Bcl}-2$, rather than Bcl$\mathrm{xL}$, contributed predominantly to the induction of AIC47-mediated autophagy. In primary chronic myeloid leukemia cells, dissociation of Bcl-2 and Beclin-1 was also observed in WT-BCR-ABL cells, whereas binding remained in T315I-BCR-ABL cells (Online Supplementary Figure $S 7 A$ ). The knockdown of $\mathrm{Bcl}-2$ induced autophagy and decreased BCR-ABL expression even in T315I-BCRABL cells (Figure $2 \mathrm{~B}$ ). Overexpression of Bcl-2 by using a pIRESneo-Bcl-2 vector ${ }^{9}$ reversed the expression of $\mathrm{BCR}$ $\mathrm{ABL}$ (Figure 2C). A Bcl-2 inhibitor, ABT-737, induced dissociation of $\mathrm{Bcl}-2 / \mathrm{Beclin}-1$ and autophagic degradation of both WT- and T315I-BCR-ABL; these effects were enhanced in combination with AIC-47 (Online Supplementary Figure $S 7 B$ ). These results suggested that $\mathrm{Bcl}-2$ was one of the essential molecules for autophagic degradation of $\mathrm{BCR}-\mathrm{ABL}$. We also investigated the MAPK-mediated phosphorylation of Bcl-2. AIC-47 decreased $\mathrm{Bcl}-2$ expression; whereas the ratio of phosphorylated Bcl-2 was increased in WT-BCR-ABL cells (Figure 2D). After treatment with AIC-47, the level of phosphorylation of JNK was decreased in WT-BCR-ABL cells; however, the level of phosphorylation of Erk was increased in these cells (Figure 2D). A MEK inhibitor decreased the phosphorylation of $\mathrm{Bcl}-2$ and disrupted the AIC-47-mediated autophagy flux (Online Supplementary Figure S8). These results suggested that Erk-mediated phosphorylation of Bcl-2 also contributed to the induction of autophagy by AIC-47 and that inactivation of MAPK/Erk signaling was one of the causes of impaired autophagy in T315I-BCR-ABL cells. We speculated that some upstream kinases might contribute to the difference in phosphorylation levels. We, therefore, used gene set enrichment analysis, which revealed that Ras-related signaling was significantly upregulated in WT-BCR-ABL cells (Figure 2E). Previous experiments demonstrated that Ras/MAPK signaling promotes autophagic cell death through the NOXA/Beclin-1 pathway. ${ }^{10}$ Ras functions as a molecular switch by cycling between an active GTP-bound form and an inactive GDP-bound form. Interestingly, the amount of GTPbound Ras was less in T315I-BCR-ABL cells than in WTBCR-ABL cells (Figure 2F), indicating that the activation of Ras was associated with the difference in activation of MAPK/Erk signaling. However, further investigation is needed.

The increased stability of T315I-BCR-ABL protein was expected to result in a cell growth advantage. Rapamycin $(0.5 \mathrm{nM})$ significantly inhibited cell growth in WT-BCRABL cells, whereas inhibition was barely observed in T315I-BCR-ABL cells (Online Supplementary Figure S9). The dissociation of $\mathrm{Bcl}-2 / \mathrm{Beclin}-1$ by treatment with Z36 or silencing of $\mathrm{Bcl}-2$ induced growth inhibition in both WT- and T315I-BCR-ABL cells (Online Supplementary Figure S9), but this effect was more marked in the T315IBCR-ABL cells. These results partly supported our contention that the autophagic degradation of BCR-ABL might be related to cell growth. However, this study has limitations to elucidate the association between autophagy and cell growth completely. AIC-47 could have multiple targets other than autophagic signaling and exhibit anti-leukemic effects even in T315I-BCR-ABL cells. Autophagy is intimately connected with apoptosis, as the same regulators, such as Bcl-2, can control both autophagy and apoptosis. ${ }^{11}$ Rapamycin $(1 \mathrm{nM})$ could also inhibit cell growth by induction of apoptosis (Online Supplementary Figure S9). Further studies are needed to reveal the association between autophagic degradation of BCR-ABL protein and cell growth.

Autophagy is essential for the maintenance of cellular homeostasis in response to various stress conditions. Depending on the circumstances, autophagy can either promote or inhibit cell survival. Several groups reported that autophagy regulates leukemogenesis ${ }^{12}$ and that pharmacological inhibition of autophagy enhances the effects of tyrosine kinase inhibitors. ${ }^{13}$ There is opposing evidence with respect to the tumor-inhibiting effects of autophagy, which showed that BCR-ABL acts as a suppressor of autophagy. ${ }^{14}$ The anti-leukemic effects of arsenic trioxide are also caused by autophagic degradation of WT-BCR-ABL. ${ }^{15}$ Our data indicate that the dissociation of Bcl-2 from Beclin-1 was impaired in T315IBCR-ABL cells, resulting in the impaired autophagic degradation that contributed to the extension of the halflife of T315I-BCR-ABL (Figure 2G). Although inhibition of autophagy is effective at enhancing the sensitivity of tyrosine kinase inhibitors to BCR-ABL, it could also contribute to increasing the stability of BCR-ABL. Our findings suggest that there is still room for argument about autophagy-targeting cancer therapy.

Haruka Shinohara, ${ }^{1}$ Yosuke Minami, ${ }^{2,3}$ Tomoki Naoe ${ }^{4}$ and Yukihiro Akao

'United Graduate School of Drug Discovery and Medical Information Sciences, Gifu University, 1-1 Yanagido; 'Department of Hematology, National Cancer Center Hospital East, Chiba; ${ }^{3}$ Department of Transfusion Medicine and Cell Therapy, Kobe University Hospital and ${ }^{4}$ National Hospital Organization Nagoya Medical Center, Naka-ku, Japan

Acknowledgments: we thank Dr. Brian J. Druker and Dr. Yuko Sato for giving us the cell lines used.

Funding: this work was supported by grant-in-aid for a research fellow from the Japan Society for the Promotion of Science (JSPS; $16 J 08131$ to HS), grant-in-aid for Scientific Research from the JSPS 
(JP18K15273 to HS), and grant-in-aid for scientific research from the Ministry of Education, Science, Sports, and Culture of Japan

(25293218 to TN)

Correspondence: HARUKA SHINOHARA.

harukashinohara313@gmail.com

doi:10.3324/haematol.2018.194431

Information on authorship, contributions, and financial \& other disclosures was provided by the authors and is available with the online version of this article at wWw. haematologica.org.

\section{References}

1. Shinohara $\mathrm{H}$, Taniguchi $\mathrm{K}$, Kumazaki M, et al. Anti-cancer fatty-acid derivative induces autophagic cell death through modulation of PKM isoform expression profile mediated by bcr-abl in chronic myeloid leukemia. Cancer Lett. 2015;360(1):28-38.

2. Shinohara H, Kumazaki M, Minami Y, et al. Perturbation of energy metabolism by fatty-acid derivative AIC-47 and imatinib in BCRABL-harboring leukemic cells. Cancer Lett. 2016;371(1):1-11.

3. La Rosee P, Johnson K, Corbin AS, et al. In vitro efficacy of combined treatment depends on the underlying mechanism of resistance in imatinib-resistant Bcr-Abl-positive cell lines. Blood. 2004;103(1):208215.

4. Kano Y, Akutsu M, Tsunoda S, et al. In vitro cytotoxic effects of a tyrosine kinase inhibitor STI571 in combination with commonly used antileukemic agents. Blood. 2001;97(7):1999-2007.

5. Tasdemir E, Maiuri MC, Tajeddine N, et al. Cell cycle-dependent induction of autophagy, mitophagy and reticulophagy. Cell Cycle. 2007;6(18):2263-2267.

6. Kang R, Zeh HJ, Lotze MT, Tang D. The Beclin 1 network regulates autophagy and apoptosis. Cell Death Differ. 2011;18(4):571-580.

7. Levine B, Sinha S, Kroemer G. Bcl-2 family members: dual regulators of apoptosis and autophagy. Autophagy. 2008;4(5):600-606.

8. Lin J, Zheng Z, Li Y, et al. A novel Bcl-XL inhibitor Z36 that induces autophagic cell death in Hela cells. Autophagy. 2009:5(3):314-320.

9. Akao Y, Maruyama W, Shimizu S, et al. Mitochondrial permeability transition mediates apoptosis induced by $\mathrm{N}$-methyl(R)salsolinol, an endogenous neurotoxin, and is inhibited by $\mathrm{Bcl}-2$ and rasagiline, $\mathrm{N}$ propargyl-1(R)-aminoindan. J Neurochem. 2002;82(4):913-923.

10. Elgendy M, Sheridan C, Brumatti G, Martin SJ. Oncogenic Rasinduced expression of Noxa and Beclin-1 promotes autophagic cell death and limits clonogenic survival. Mol Cell. 2011;42(1):23-35 .

11. Gordy C, He YW. The crosstalk between autophagy and apoptosis: where does this lead? Protein Cell. 2012;3(1):17-27.

12. Altman BJ, Jacobs SR, Mason EF, et al. Autophagy is essential to suppress cell stress and to allow BCR-Abl-mediated leukemogenesis. Oncogene. 2011;30(16):1855-1867.

13. Bellodi C, Lidonnici MR, Hamilton A, et al. Targeting autophagy potentiates tyrosine kinase inhibitor-induced cell death in Philadelphia chromosome-positive cells, including primary CML stem cells. J Clin Invest. 2009;119(5):1109-1123.

14. Sheng Z, Ma L, Sun JE, Zhu LJ, Green MR. BCR-ABL suppresses autophagy through ATF5-mediated regulation of MTOR transcription. Blood. 2011;118(10):2840-2848.

15. Goussetis DJ, Gounaris E, Wu EJ, et al. Autophagic degradation of the BCR-ABL oncoprotein and generation of antileukemic responses by arsenic trioxide. Blood. 2012;120(17):3555-3562. 